\title{
Moving online: Experiences and potential benefits of digital dance for older adults and individuals with Parkinson's disease
}

\author{
Judith Bek ${ }^{1,2}$, David Leventhal ${ }^{3}$, Michelle Groves ${ }^{4}$, Charlotte Growcott ${ }^{2}$, Ellen Poliakoff ${ }^{2}$ \\ ${ }^{1}$ School of Psychology, College of Social Sciences and Law, University College Dublin, Ireland \\ ${ }^{2}$ Division of Neuroscience and Experimental Psychology, School of Biological Sciences, University \\ of Manchester, United Kingdom \\ ${ }^{3}$ Mark Morris Dance Group - Dance for PD, Brooklyn, NY, USA \\ ${ }^{4}$ Faculty of Education, Royal Academy of Dance, London, United Kingdom
}

Corresponding author: Judith Bek, School of Psychology, College of Social Sciences and Law, University College Dublin, Belfield, Dublin D4, Ireland. judith.bek@ucd.ie

\begin{abstract}
Background. Dance is found to provide a range of beneficial effects for older adults including individuals with age-related neurological conditions such as Parkinson's disease (PD). The COVID-19 pandemic accelerated the development of at-home dance programs delivered digitally through live and pre-recorded media, but little is known about how participants may engage with and benefit from these resources.

Objective. This study explored experiences and potential benefits of digital dance resources among healthy older adults and individuals with neurological conditions.

Methods. An online survey consisting of fixed-choice and open questions was designed in collaboration with dance program providers and distributed between June and November 2020.

Results. High levels of engagement in at-home dance programs were found among healthy older adults $(\mathrm{N}=$ 149) and individuals with PD $(\mathrm{N}=178)$. Sensorimotor outcomes (e.g., balance, posture) were more widely reported among individuals with PD, while older adults reported similar numbers of sensorimotor and nonmotor (e.g., mood, confidence) outcomes. The use of strategies (imagery and vocalising) during participation were differentially associated with outcomes in older adults and PD groups. At-home dance was found to offer convenience and flexibility, but participants missed the interaction, support and routine of in-person classes. The majority expressed a preference to continue with both digital and in-person participation in the future. Qualitative analysis of participants' comments reinforced the quantitative findings, while also revealing that online dance could help to maintain connection and well-being, and identifying further considerations for improving accessibility and facilitating digital engagement.
\end{abstract}

Conclusions. At-home dance programs appear to be accessible and engaging for older adults, including individuals with PD, although potential barriers to participation need to be addressed. Digital resources for home-based activities will be increasingly important to enable cost-effective, large-scale provision of therapeutic activities for older adults.

Keywords: Parkinson's disease; ageing; COVID-19; digital health; dance and movement. 


\section{Introduction}

With the world's ageing population, the prevalence and economic burden of age-related health conditions such as Parkinson's disease (PD) is increasing [1]. The physical inactivity of many older adults [2] is associated with a range of poor health outcomes [3], and people with PD are around one-third less physically active in comparison to the general population [4]. There is clearly a critical need to identify and develop sustainable methods to increase levels of activity, independence and well-being in older adults and those with conditions such as PD.

The contribution of creative approaches to health promotion and rehabilitation is increasingly recognised, as indicated by a recent review of the evidence on arts and health by the World Health Organization [5]. Dance has received particular attention from health professionals and researchers as a form of activity that may provide an engaging and beneficial option for older people, and quantitative and qualitative studies have demonstrated a range of promising effects. Systematic reviews and meta-analyses have documented evidence that dance may reduce the risk of falls and increase strength, balance and mobility in older adults [6-8], as well as improving non-motor outcomes including cognitive function [9-11] and psychosocial well-being [10,11]. The potential therapeutic benefits of dance for people living with age-related neurological conditions, particularly PD, as well as others such as stroke and dementia, have also been investigated. Reviews have highlighted evidence for improvements in motor symptoms, functional mobility, and cognition in people with PD [12-14], while indicating the need for further evidence in relation to non-motor outcomes [15].

Although dance involves physical exercise, the complexity of dance as a motor, cognitive, emotional and social activity likely contributes to its positive outcomes [16,17], and dance has been associated with enhanced neural plasticity when compared with repetitive exercise in older adults [18]. Importantly, older people and individuals with PD show high levels of adherence to dance $[8,14,17]$, indicating its potential to provide an effective and sustainable approach.

The restrictions in social contact and participation imposed in response to the COVID-19 pandemic significantly impacted older people, many of whom faced the highest levels of isolation because of the agerelated risk factors associated with the virus [19,20]. In these circumstances, the importance of digital alternatives for maintaining communication and activity was brought to the forefront. Older people are increasingly confident in using digital technology, and studies during the pandemic found that digital tools were used by older adults to maintain social connections [21], as well as by people with PD to engage in physical activity [22,23]. Nonetheless, the level of technology use among older adults is likely still lower than in other age groups [24], and so their use of online social and health resources may be more limited.

Following the suspension of in-person group activities during the pandemic, providers of community dance programs, including initiatives designed specifically for older adults and individuals with PD, quickly transitioned to online classes. Considering the potential barriers relating to the use of technology, and the differences between in-person classes and online participation (such as levels of social contact and instructor feedback), the present study used an online survey to investigate users' experiences and perceived benefits of these new resources.

The survey explored access to and engagement with digital home-based dance programs among older adults, as well as the potential outcomes of these programs across sensorimotor and non-motor domains. For individuals with neurological conditions like PD, there may be additional barriers or challenges to participation, such as difficulties with motivation [25] or concerns about safety [26], or they may be less able to experience benefits of dance without the support and guidance provided within group classes. The present study therefore also sought to identify any differences in the types of benefits experienced by neurologically healthy older adults and those with PD, as well as factors that may influence outcomes for each user group. 
Initial findings of the survey based on data collected from individuals with PD [27] indicated that homebased dance programs were accessed with high levels of engagement, and revealed a range of potential sensorimotor and non-motor benefits. This initial analysis also found that perceived benefits were greater among participants engaging in particular cueing strategies such as imagery during dance classes. The present study further examines such mechanisms of engagement and their relationship with potential benefits, in older adults without neurological conditions and those with PD. Qualitative analysis was also conducted to explore participants' experiences in greater depth.

\section{Methods}

An online survey was designed in collaboration with providers of community dance programs for older adults and people with PD (Silver Swans ${ }^{\circledR}$ - Royal Academy of Dance; Dance for PD ${ }^{\circledR}$ - Mark Morris Dance Group), with additional input from dance instructors, physiotherapists, and individuals with PD who had previously participated in dance.

The survey consisted primarily of fixed-choice questions addressing access to and use of digital dance programs, including engagement in cueing strategies during dance (different types of imagery; vocal and rhythmic cues), perceived benefits, advantages and disadvantages of at-home participation, and views on future participation. Two optional open-ended questions were also included to allow participants to provide further information if they wished ("Do you have any suggestions for how at-home dance resources could be improved?"; "Is there anything else you would like to tell us about your experience of dance at home resources?"). The survey was also open to individuals who were interested but not currently participating in dance, who were directed to a subset of questions addressing previous participation, barriers to accessing resources, and interest in future participation.

The survey was administered using SelectSurvey.net (v4.033.002; ClassApps, Overland Park, KS, USA). Invitations to participate in the survey were distributed between June and November 2020 through dance organisations and practitioners, community groups, research volunteer lists, and social media. The study was approved by the University of Manchester Research Ethics Committee and all participants provided informed consent via an online form prior to accessing the survey.

\section{Data analysis}

Since only $3.4 \%$ participants reported a neurological condition other than PD, and a further $1.1 \%$ did not report whether or not they had a neurological condition, the analysis reported here focuses on older adults without a neurological condition (OA) and individuals with PD.

Responses to fixed-choice questions were summarised as percentages and are reported descriptively in the following section. Statistical analyses were conducted to further examine outcomes of participation in the two main respondent groups (OA and PD). Twelve items from a checklist of potential outcomes of dance participation were categorised as either sensorimotor or non-motor (6 items each), and were then compared using a group (OA, PD) x type (sensorimotor, non-motor) ANOVA. To explore how the use of particular strategies within at-home dance participation might be associated with reported benefits, linear mixed effects modelling was conducted in $\mathrm{R}$ [28] using the package lme4 [29]. This approach enables the effects of fixed factors (predictors) to be analysed while adjusting for random effects arising from individual variation between participants. Responses to the two open-ended questions were analysed using an inductive/datadriven thematic approach [30]. Two of the authors (CG and JB) independently coded the responses, using Microsoft Excel and NVIVO to identify and categorise codes. Candidate themes were generated, which were then discussed further with a third author (MG) before being finalised. 


\section{Results}

Valid survey responses were collected from 466 individuals, including 276 people living with PD and 16 with other neurological conditions, as well as 5 who did not report whether or not they had a neurological condition. Respondents were aged between 43 and 89 years, and the majority were from the United Kingdom $(72.1 \%)$ or USA $(20.4 \%)$.

\section{Experiences and perceptions of accessing and using at-home dance resources}

The characteristics of respondents who were currently using at-home dance programs $(\mathrm{N}=351)$ are summarised in Table 1. The length and frequency of participation in at-home dance programs was similar between OA and PD respondents: the majority had been dancing at home for less than 12 months, and most were currently participating at least once a week. Nearly all of the OA respondents (95.3\%) and $78.7 \%$ of the PD respondents had previously attended in-person classes.

Table 2 summarises the responses to questions exploring access to digital resources, use and barriers, preferences in the selection and use of resources, and considerations for future provision. Results are summarised separately for older adults with no neurological condition (OA) and those with Parkinson's (PD), in addition to the overall results.

Table 1. Characteristics of respondents using home-based resources summarised for all users of at-home dance programs, older adults (OA), and individuals with Parkinson's (PD)

\begin{tabular}{llll}
\hline & All users $(N=351)$ & $O A(N=149)$ & $P D(N=178)$ \\
Age (years: M; range) & $66.9 ; 43-88$ & $66.0 ; 50-89$ & $69.5 ; 47-88$ \\
Gender (\% female; male) & $83.2 ; 14.5$ & $94.6 ; 4.7$ & $75.8 ; 24.2$ \\
$\begin{array}{l}\text { Previous in-person dance } \\
\text { participation (\% yes) }\end{array}$ & 85.2 & 95.3 & 78.7 \\
$\begin{array}{l}\text { Frequency of at-home practice } \\
\text { (\%): }\end{array}$ & & \\
Less than once weekly & 5.7 & & \\
Once weekly & 30.5 & 6.7 & 5.1 \\
Twice weekly & 31.6 & 32.2 & 29.2 \\
More than twice weekly & 29.6 & 36.2 & 28.1 \\
Length of participation (\%): & & 24.2 & 36.5 \\
0-3 months & 37.3 & & \\
3-6 months & 26.8 & 48.3 & 29.2 \\
6-12 months & 13.4 & 28.2 & 26.4 \\
$>$ 12 months & 15.1 & 8.1 & 18.5 \\
Use of other resources for home- & 64.7 & 12.8 & 16.9 \\
based activities (\% yes) & & 53.7 & 77.5 \\
Use of online platforms/media & 74.4 & & 73.6 \\
for social interaction (\% yes) & & 83.2 & \\
\hline
\end{tabular}


Table 2. Survey responses (\%) summarised for all users of at-home dance programs, older adults (OA), and individuals with Parkinson's (PD). The most frequent responses to each item are indicated in bold for each group.

All users $O A \quad P D$

Media type used:

Live-streamed class

Interactive online class (e.g., Zoom)

Pre-recorded online class

DVD

Other (e.g., printed instruction)

Preferred media:

Live

Pre-recorded

Both live and pre-recorded

No preference

Factors influencing choice of resources:

Dance style (e.g., ballet, modern, mixed)

Familiar program/instructor

Free classes only

Low cost

Scheduled classes

Opportunities for social connection

Reputation/brand

Recommendation

Difficulty level

Type of media/platform

Difficulties in accessing or using resources:

Connectivity/network problems

Setting up or using software

Image quality

Sound quality

No problems

Aspects missed from in-person participation:

Interaction with the instructor

Interaction with others

Support/encouragement

Live music

Social activities before/after class

Plans for future participation:

Attend in-person classes only

Continue with home practice only

Both attend classes and continue with home practice

Neither

Interest in educational resources to supplement practice:

Written information

Educational video

Both written information and video

Neither

$\begin{array}{lll}\mathbf{6 3 . 5} & \mathbf{6 3 . 1} & 66.3 \\ 49.6 & 55.0 & 47.2 \\ 62.7 & 57.7 & \mathbf{6 9 . 1} \\ 17.1 & 1.3 & 28.7 \\ 1.1 & 0.7 & 1.1\end{array}$

64.0

45.8

64.5

19.8

15.0

18.7

21.8

28.0

11.2

3.6

1.9

3.7

68.4

79.9

60.1

57.3

61.7

55.6

27.1

32.0

35.6

23.5

39.9

53.4

53.7

30.9

29.1

27.5

34.3

37.6

34.2

32.9

41.6

37.6

40.2

43.6

35.9

7.7

42.2

29.2

8.8

8.1

7.3

20.5

10.7

6.7

15.2

50.4

27.5

61.8

69.5

77.2

63.5

77.8

89.3

$\mathbf{7 0 . 8}$

44.7

51.7

30.2

38.8

31.1

31.5

43.3

47.0

40.5

18.2

9.7

24.2

12.4

68.4

6.0

69.1

13.5

0.3

0.0

70.8

0.6

8.9

8.7

9.6

11.1

8.7

47.0

13.5

51.6

34.9 
(Table 2 continued)

All users

$O A$

$P D$

Interest in trying new technology-based approaches to dance:

Practicing dance movements using an app

20.8

9.4

22.8

19.1

Virtual reality dance

44.2

8.7

9.0

Both app and virtual reality

23.4

37.6

51.7

Neither

30.2

19.1

Both live and pre-recorded classes were widely used, although the use of DVDs was more common among respondents with PD, possibly reflecting the availability of at-home DVDs from the Dance for PD® program preceding the pandemic. Among those using both live and recorded media, there was a preference for live classes, although this was more evident in the data from participants with PD. The most important factor in choosing at-home programs was the style of dance, followed by familiarity and scheduled classes. Half of all participants experienced some technical difficulties when accessing or using resources, most commonly involving connectivity/network problems, although OA respondents reported more difficulties than PD respondents.

The reported advantages and disadvantages of home-based dance participation are illustrated in Figure 1, which shows a broadly similar pattern of results between groups, although OA respondents tended to report disadvantages more frequently and advantages less frequently than those with PD. In both groups, the most commonly reported advantages were not having to travel, flexible timing, and ease of access enabling more frequent participation. The greatest disadvantages were the lack of social interaction and one-to-one support, and reduced motivation resulting from the loss of routine.
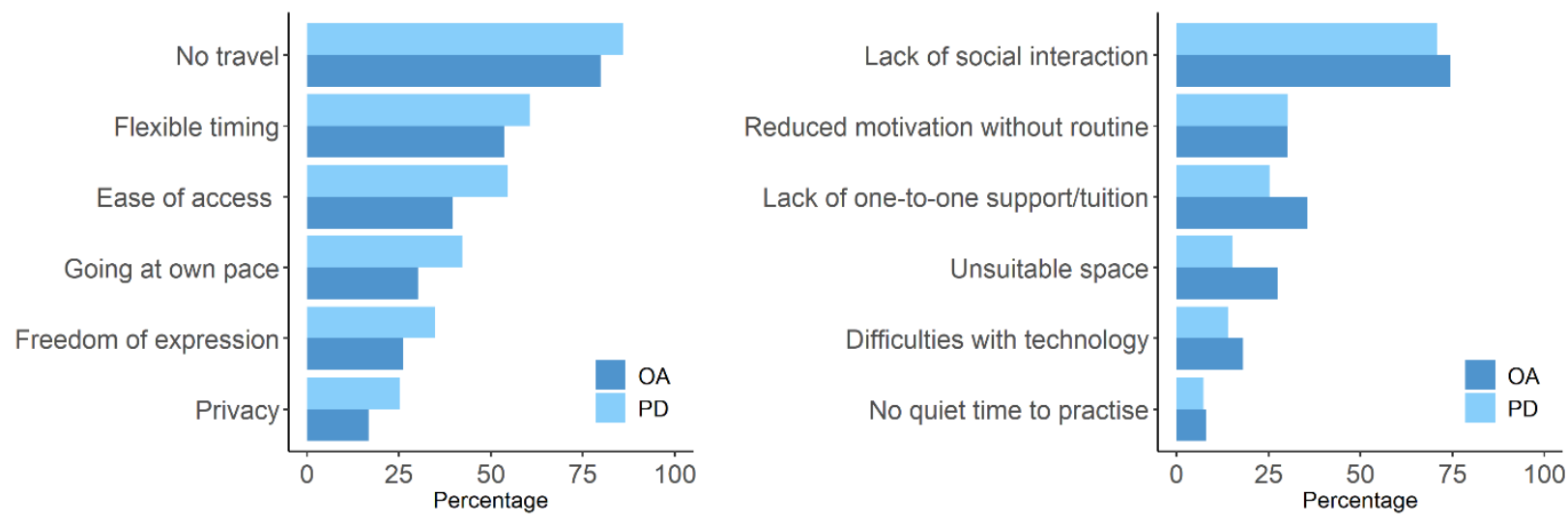

Figure 1. Advantages (left) and disadvantages (right) of at-home dance participation reported by older adults (OA) and individuals with PD.

Participants previously attending in-person classes primarily missed the interaction with other participants and instructors, and these aspects were more frequently noted by OA than PD respondents. Nonetheless, the data clearly indicated a desire among both groups to continue participating in at-home classes alongside inperson classes when these resumed. The majority of participants were also open to receiving written and visual resources to supplement their practice, as well as using apps or virtual reality for dance in the future. 


\section{Reported benefits and strategies}

As shown in Figure 2, both OA and PD respondents reported a range of sensorimotor benefits (e.g., improved posture and balance, greater ease of movement) and non-motor benefits (e.g., improved mood, reduced stress/anxiety, increased energy). Only 6\% of OA respondents and 5.1\% of PD respondents did not perceive any benefits of at-home participation.

Statistical analysis found no significant effect of group on the overall number of benefits reported $(F(1,312)$ $\left.=1.89 ; p=.17 ; \eta^{2} G=.0045\right)$, but there was a significant effect of type $\left(F(1,312)=3.99 ; p=.047 ; \eta^{2} G=\right.$ $.0033)$, and a significant group*type interaction $\left(F(1,312)=10.02 ; p=.0017 ; \eta^{2} G=.0082\right)$. As illustrated in Figure 2, pairwise comparisons revealed that individuals with PD reported significantly more sensorimotor than non-motor benefits (mean difference $=.53 ; p=.0009$ ), while OA respondents reported similar numbers of sensorimotor and non-motor benefits (mean difference $=.12 ; p=1.0$ ).
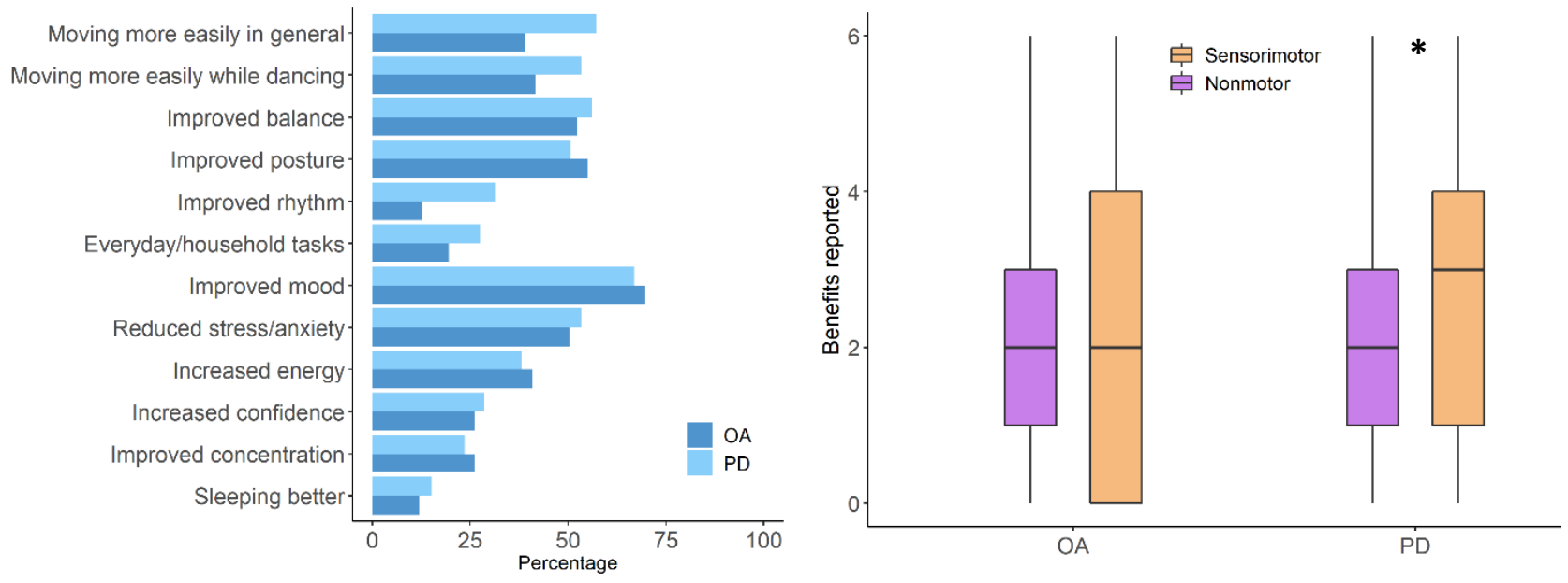

Figure 2. Positive outcomes of at-home dance reported by older adults (OA) and individuals with PD. The bar chart (left) shows the percentage of participants in each group reporting each outcome, and the boxplot (right) shows medians and quartiles for sensorimotor and non-motor outcomes in each group. A greater number of sensorimotor benefits were reported by participants with PD, while OA respondents reported similar numbers of both sensorimotor and non-motor benefits. ${ }^{*} p<.001$.

Figure 3 shows the reported use of strategies and cues during at-home dance participation. The relationship between strategy use and perceived benefits within each group was explored using linear mixed-effects modelling. A baseline model included the intercept for participants as a random effect and outcome type (sensorimotor vs. non-motor) as a fixed factor. To determine the potential influence of specific aspects of engagement, the following strategies and their interactions with outcome type were entered into the model for each group: counting, vocalising, singing, visual imagery, kinesthetic imagery, and analogy/metaphor imagery. Two further items, "watching the instructor closely" and "playing my own music in the background", were omitted from analysis due to the respectively very high and low percentages of participants endorsing these strategies. To identify which strategies contributed significantly to the prediction of reported benefits, one item at a time was removed from subsequent versions of the model, and likelihood ratio tests were used to compare each model with the previous more complex one. 

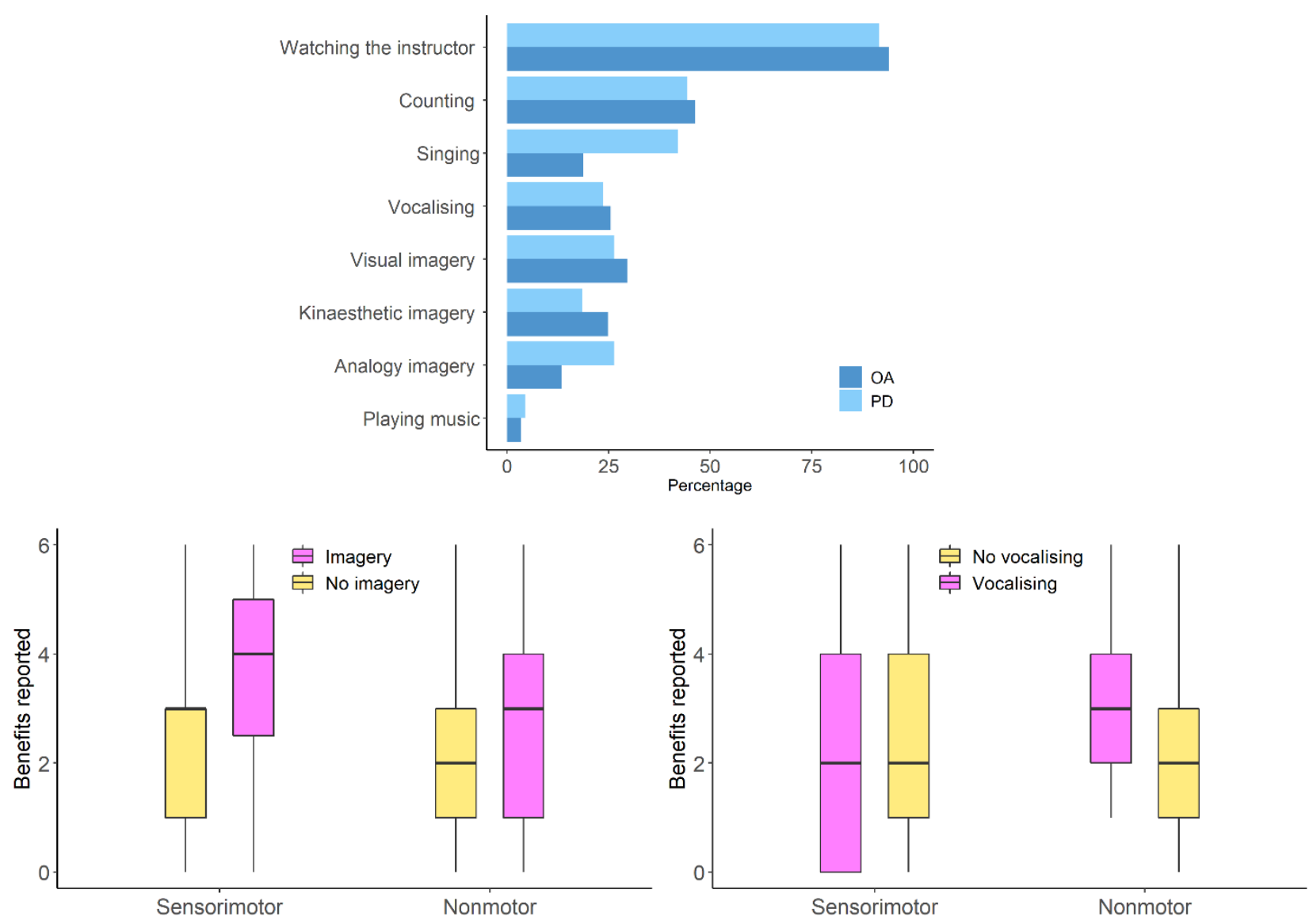

Figure 3. Strategies used while participating in at-home dance. The upper panel shows the percentage of participants in each group reporting the use of each type of strategy. The lower panel show numbers of benefits reported (median and quartiles) according to strategy use: in individuals with PD (left) sensorimotor benefits were associated with the use of analogy/metaphor imagery, and in OA respondents (right) nonmotor benefits were associated with vocalising.

For OA participants, likelihood ratio tests showed that the use of vocalising $\left(\chi^{2}(2)=7.49 ; p=.0002\right)$ and visual imagery $\left(\chi^{2}(2)=6.08 ; p=.048\right)$ contributed significantly to the fit of the model. There was also a significant interaction between vocalising and benefit type $(b=1.023, S E=0.403, t(142)=2.54 ; p=.011)$, showing a specific association with non-motor benefits. For PD participants, visual imagery $\left(\chi^{2}(2)=9.92 ; p\right.$ $=.007)$ and analogy/metaphor imagery $\left(\chi^{2}(2)=13.39 ; p=.001\right)$ contributed significantly to the model, and there was a significant interaction effect between analogy/metaphor imagery and benefit type $(b=-0.633$, $S E=0.288, t(172)=-2.195 ; p=.028)$, indicating a specific association with sensorimotor benefits. Comparisons between models indicated that outcomes in the OA group were best predicted by both vocalising and visual imagery, while outcomes in the PD group were best predicted by the use of analogy/metaphor imagery.

\section{Qualitative analysis}

Thematic analysis did not identify any clear differentiation between OA and PD respondents in the content of open comments, so themes were generated across the combined OA and PD data. The themes are described in Table 3 with illustrative quotes. 
Table 3. Themes generated from open comments of older adults (OA) and individuals with PD (PD).

Theme 1. Maintaining connection and well-being.

This theme reinforced findings from the fixed-choice questions, indicating that participants missed the social connection from in-person classes.

"Nothing replaces actually being in the dance studio with a teacher and other dancers" [OA]

"Although I myself am enjoying them at present, I still prefer real life classes, with all their interaction" [PD]

"I find the online classes frustrating (though wonderful) in a way...we are missing the expressions of the other students, the subtleties of personal expression, the smiles, the nods, the ability to work in pairs or groups and separate out to create" [OA]

However, a sense of community could be found through connecting with others online in live classes.

"You feel connected to the rest of the group even if it's done remotely" [OA]

“...feel I am part of something even though no-one else is there!” [PD]

Being able to access at-home dance programs provided participants with a structure to the week and a means of maintaining their physical and mental health during COVID-19 restrictions.

"They do enable us to...protect our state of mind and maintain a level of fitness that would otherwise diminish" [OA]

"The online Zoom class the teacher offered and the discovery of online pre-recorded classes has helped with coping with the lockdown restrictions on my life" [OA]

"It really has added structure to my week. I feel as though I have met new friends. What could have been better during this period of lockdown and global pandemic. I do think it is of paramount importance that people of my age continue to do the things they love, to keep them fit and healthy and in a good place mentally." [OA]

"I am really enjoying dance at home and feel much better physically and mentally after a class. It is especially beneficial at this time, when there are so many restrictions." [PD]

One participant also noted that practising at home helped to maintain confidence for returning to regular group sessions.

"It also gives me confidence in my ability when I go to live classes" [OA]

Many respondents simply expressed gratitude for the adaptations made by instructors and organisations that provided the opportunity to continue dancing.

"I feel extremely fortunate to have been able to follow classes online" [OA]

"They have been a life saver especially during this troubling time" [PD]

Theme 2. Advantages and opportunities in online participation.

This theme also reiterated the numerical responses by highlighting advantages of at-home participation, such as the convenience of not having to travel, the capacity to dance more frequently, and the provision of a safe space in which to move and express oneself without judgement.

"In several ways I have found this preferable to actually being in class (not having to travel...)" [OA]

“Please continue after COVID-19. No traveling to class huge benefit!!!” [PD]

"I have been practising regularly and find my balance in particular is very much improved... I must confess that before lockdown the only time I danced was in class once a week with no practice in between." [OA]

"The number of times each week it allows me to dance is terrific. I love dancing every day - sometimes 
morning and afternoon." [PD]

"The online experience of classes enables me to choose (having tried several different ones) which one I feel I am able to do that day and at a time convenient to me." [OA]

"Allows people who are physically restricted to move freely and use their creativity and imagination without being judged" [PD]

The desire to continue with online classes was also echoed.

"I have enjoyed the dance experiences and intend to continue in class and online in the future" [OA]

"Please keep this available as I live in a small town with 450 people and no activity like this offered" [PD]

"They are a tremendous help during the pandemic, but I shall continue using them afterwards" [PD]

"I also believe it would continue to be useful in the future for those who are unable to travel to ordinary live classes, and for those who are keen to dance as much as possible" [PD]

The switch to online participation led to new experiences and opportunities for some participants, such as the possibility of joining classes in remote locations and meeting new people online. Others had been able to try new classes or dance styles by searching online, or even discovered dance for the first time.

"I have also enjoyed some online classes found on YouTube which were taught in different ways" [OA]

"I also like trying out new classes and if I like the video, I add them on to my workout selection" [PD]

"COVID19 lockdown led me to join an online community of over 50s dancers where I was made welcome. I would never have met these dancers under normal circumstances because they are based in Yorkshire over 100 miles away from my home." [OA]

"I also like meeting dancers from all over the world. If not virtual, I would not be able to attend. A gift!" [PD]

“...the interaction with people from across the world was fascinating and motivating” [PD]

It was also noted that the learning of new technology skills had been accelerated during the pandemic, although there may have been initial challenges.

"It has made me feel more confident using video and computer technology" [OA]

"They started a Zoom class early in lockdown but I got put off when they were getting it going, and didn't realise I could highlight one dancer to follow and was trying to follow one person when I was still in gallery mode!" [PD]

Some individuals had found ways of using the digital format to create choreographies in online classes.

"It has opened up new creative pathways to explore and introduced an interesting visual perception of how other members of the group move and react to the process of creating choreography online." [OA]

Theme 3. Effectiveness of engagement, teaching and learning.

This theme encapsulated both positive and negative aspects of at-home participation, and elements that respondents considered important for optimising or improving the experience.

It was noted that it could be difficult to maintain motivation, although scheduled classes helped to encourage regular participation.

"It's very convenient but you have to be self motivated" [PD]

"I find having a regular time slot enables me to plan for it" [PD]

"It is very difficult to keep myself motivated. It is the classes that I know I will return to after lockdown at some point that keep me going. " [OA] 
"It was good to be able to keep the connection with the weekly class as it helps motivate a structure in the day at a specific time." [OA]

It was also suggested that smaller classes with a regular group would be preferable.

"Teachers should maybe keep a class small and there should be a registration so you are in an actual group like a real class without so much come and go of the students" [OA]

"Smaller classes so some personal feedback possible" [OA]

The importance of having instructors who were experienced in working with a particular group and sensitive to the needs of participants was highlighted. Familiarity with the instructor also appeared to increase motivation and engagement.

"Our teacher takes into consideration actions we may be unable to do at our age. She is good at breaking down each routine into smaller 'bites'." [OA]

"I like the contact with my regular teacher and understand her way of teaching" [OA]

"We have an excellent teacher who is very good at explaining the steps. This makes our on line classes very enjoyable. If our teacher was not as good, this might be a problem." [OA]

"The teacher ... uses music as background sound but does not coordinate the rhythm and the movements or consider the effect of the tempo on the movement. My movement is more fluid if I have appropriate external cues." [PD]

"I love the enthusiasm of the teachers" [PD]

Some individuals found it difficult to follow the instructor's movements if a reversal of directions was required. Having a clear visual demonstration (including appropriate camera angles) was also valued.

"I have difficulty following in mirror image, muddled with rights and lefts" [OA]

"Demonstrations of the steps using more than one camera so we can see different angles" [OA]

"One thing we really value in the at-home dance resources is when instructors are very visible and both describing and doing the movements when showing us a new segment" [PD]

Another limitation was that the home environment could be restrictive or unsuitably equipped for dance.

"It would be great if it were possible to more easily purchase supplies for use at home -- e.g., we have a carpeted living room, and that is terrible for ballet. A suitable mat that could be rolled up and kept out of the way" [OA]

"The space I have at home is not sufficient to do more than a barre and limited exercises in the centre" [OA] "I find space restricts me - I like to really MOVE and travel while dancing" [PD]

The need to make online classes easier to access was noted, such as through the improvement of technical knowledge and provision of guidance on how to find suitable resources.

"Training for both instructors and users on how best to use videoconferencing" [PD]

"Providing a directory or central portal where resources, classes etc. could be located" [OA]

Other comments expressed the need for greater diversity in provision, to facilitate participation across different locations, languages, and cultures.

"Could consider live participation and schedule more classes for other time zones" [PD]

"More classes in other languages... I often think about all the other people that may not participate because of language barriers. In general too, I think the at-home resources need to increase advertising for racial and ethnic minorities because it's a very homogeneous group that participates." [PD] 
Theme 4: Different preferences.

It was evident that participants varied in their preferences when engaging with at-home dance. While many preferred live classes, others valued the flexibility of having recorded sessions.

"After watching and preparing the pre-recorded exercises I found it still very important to get instructions on which mistakes to avoid and personalised feedback to correct mistakes during the live sessions. Simply watching somebody who can do it well is not enough to learn how it feels to do it right." [OA]

"The interactive sessions are great fun, the pre-recorded sessions I have greater difficulty. I like both" [OA]

"I wish there were more opportunities to access more than one weekly interactive class" [PD]

"I would like to be able to access classes in a library, to be able to repeat them if wished" [PD]

Some wanted to be able to participate in a complete and continuous session, while others preferred to have exercises broken down into manageable sections.

"I would like a continuous 30-40 minute workout so I don't need to click on each exercise" [OA]

"Thinking of prerecorded videos only, it would be ideal to have starter videos that break down the steps" [PD]

Detailed verbal descriptions of the exercises were appreciated by some participants, but others found long explanations to be off-putting and disruptive of the flow of the class.

"One thing we really value in the at-home dance resources is when instructors are very visible and both describing and doing the movements when showing us a new segment" [PD]

"I feel that an edited version (to exclude the instructional dialogue) would be perfect for me" [OA]

"Some spend too much time on instruction which makes it hard to maintain momentum" [PD]

\section{Discussion}

The present study provides initial evidence that dance programs delivered through digital media are accessible and engaging for older adults and for individuals living with PD, and may provide a range of sensorimotor and non-motor benefits. While the overall number of perceived benefits of at-home dance was similar between OA and PD respondents, individuals with PD reported a greater number of sensorimotor effects, including improvements in posture, balance, and ease of movement. This difference in perceived outcomes between groups may reflect the specific features of the programs used (e.g., PD-specific classes), or different goals and objectives for participating in dance. The latter suggestion is supported by previous qualitative research indicating that addressing motor symptoms is a priority outcome of dance among people with PD [31]. For many older adults without a long-term health condition, dance may be enjoyed primarily as a social leisure activity, with a reduced focus on physical benefits. Consistent with this idea, the present study found a greater emphasis on the importance of social interaction in dance among older adults without any neurological conditions.

Analysis of the relationship between cueing strategies utilised during dance and potential benefits revealed a further difference between participant groups. Among OA respondents, the use of visual imagery was associated with the number of benefits reported overall, while vocalising predicted the number of non-motor benefits. Our previously reported initial analysis of the data from respondents with PD [27] indicated an association between the use of imagery and overall benefits reported, while the further breakdown by outcome domains in the present study revealed a more specific association of analogy/metaphor imagery with sensorimotor benefits in individuals with PD. 
A better understanding of the involvement of cognitive-motor mechanisms within dance could help to enhance its beneficial effects. Further to the more obvious elements such as physical activity, rhythm, and social interaction, the present findings indicate the potential value of imagery as a strategy that may boost positive outcomes, particularly in the sensorimotor domain among individuals with PD. The use of narrative themes and music [32,33] could support engagement in the analogy/metaphor-type imagery that often features in dance programs for people with PD [34]. As discussed in a recent review[16], movement imagery is widely used in dance, alongside observation and imitation of movements, and these processes may activate brain networks overlapping with those involved in motor preparation and execution. Most of the participants in the present study reported attending closely to the instructor's movements, and the qualitative data indicated the importance of clear visual demonstration.

The importance of being able to connect with others through dance was clear from both quantitative and qualitative data in the present study, as reported previously[32, 35, 36]. While digital participation can only partially replicate the social environment of in-person dance, live online classes in particular could help to maintain a sense of community.

The continuation of dance programs, albeit in the virtual domain, was also noted as providing a valuable means of maintaining physical and mental health in times of isolation and anxiety during lockdown. Moreover, the qualitative data revealed that digital dance programs could have some unique advantages, as participants reported their appreciation of new opportunities such as discovering new classes or different dance styles, connecting with others in distant locations, and the accelerated learning of skills in using digital technology. This digital mode of participation also provided a safe space for individuals to practice dance without feeling self-conscious and could help to maintain confidence. There was a desire among respondents to continue participating in online dance alongside in-person classes, with an appreciation of the convenience, variety, and frequency of participation offered by digital resources.

At-home programs have clear potential to broaden access to dance and other activities, particularly for those who have difficulty travelling to classes or those in rural communities. Indeed, home-based activity programs have previously shown increased levels of adherence among older adults compared with travelling to attend sessions [37]. Nonetheless, the uptake of online dance is also dependent on the availability of, and ability to engage with, the necessary technology. While around half of participants had experienced some technical problems, these mostly involved issues with connectivity, rather than lacking knowledge of or access to digital technology. However, the responses of individuals not currently using at-home programs also indicated that many may be unaware of the availability of resources, or may need support to find and access the available options. Moreover, the nature of the present study means that the results only reflect the experiences of those who already have a certain level of digital engagement. Participants' comments also highlighted the need to increase accessibility of live online classes, by catering to different time zones and more diverse sections of the community such as different language groups, although some program providers are already working to address this [38].

Additionally, variation in preferences among participants indicates that providing alternative options, such as different levels of intensity or difficulty, may increase motivation and engagement. This is consistent with qualitative studies indicating the need for appropriate challenge in dance for people with PD [31,32,33]. High-quality instruction, and the sensitivity and experience of instructors in working with particular groups, was noted to be important (see also [33]). The availability of regular scheduled classes was also suggested to help provide structure and motivation.

Many participants expressed an interest in receiving educational resources to supplement their dance practice. Given the preliminary evidence that the use of strategies such as imagery or vocalising might enhance outcomes, these could be highlighted within educational materials. Respondents also indicated an openness to trying out new technologies for dance participation. Dance exergames have been associated with 
physical, cognitive and social benefits among older adults [39] and sensorimotor outcomes in people with PD [40], and virtual reality based dance has been found to improve balance, daily activities, and mood in PD [41]. There is potential for these technologies to further enhance the at-home dance experience for older people and those with PD. For example, the use of immersive media could facilitate engagement by enabling participants to view the instructors from different angles or focus in on particular movements, thereby supporting cognitive-motor processing.

\section{Conclusions}

Based on the present findings, key considerations for further development of at-home dance programs are summarised in Figure 4. While the development of digital resources for home-based activities was accelerated as a result of restrictions during the COVID-19 pandemic, online classes are already being integrated into ongoing programming by dance organisations [38], and their use and importance will likely continue to grow as older people increase in their knowledge and confidence to use digital technology. Moreover, although digital programs cannot entirely replace in-person activities, they can offer widely available resources to support health and well-being, with the possibility of providing benefits to individuals across geographic and cultural landscapes. Further research should aim to clarify the long-term effects of online dance programs in different outcome domains, and feedback from participants will be a valuable tool in the ongoing development of accessible and engaging dance programs.

\section{Key considerations for at-home dance programs:}

- Online provision can offer a valued alternative or supplement to in-person classes for individuals unable to travel to classes or wishing to practice more frequently

- Live scheduled classes can provide social connection and motivation, particularly when attended by a regular group

- Individual preferences may be addressed by increasing variety (e.g., intensity and difficulty of classes)

- High quality teaching and individualised feedback are important

- The use of strategies such as imagery and vocalising may enhance outcomes

- Supplementary educational resources could also help to optimise the benefits of dance

Figure 4. Key considerations for the design of at-home dance programs as indicated by quantitative and qualitative data from the survey. 


\section{Acknowledgements:}

The authors thank Aline Arakaki, Anne-Marie Booth, Gayathri Ganapathy, Helen Gould, Rachel Johnston, and Matthew Sullivan, for input into the survey design, and Anam Bhutta for assistance with qualitative analysis. We also thank Parkinson's UK and the Manchester Institute for Collaborative Research on Ageing for their support with recruitment and promotion of the project.

\section{Declarations of interest:}

DL is a full-time employee of the Mark Morris Dance Group, which administers the Dance for PD® program. MG is a full-time employee of the Royal Academy of Dance and is involved in the development of its Silver Swans® initiative. The remaining authors have no conflicts of interest.

\section{References}

1. Dorsey RE, Elbaz A, Nichols E, Abd-Allah F, Abdelalim A, Adsuar JC, et al. Global, regional, and national burden of Parkinson's disease, 1990-2016: a systematic analysis for the Global Burden of Disease Study 2016. Lancet Neurol. 2018;17:939-53.

2. Gomes M, Figueiredo D, Teixeira L, Poveda V, Paúl C, Santos-Silva A, et al. Physical inactivity among older adults across Europe based on the SHARE database. Age Ageing. 2017;46:71-7.

3. Cunningham C, O' Sullivan R, Caserotti P, Tully MA. Consequences of physical inactivity in older adults: A systematic review of reviews and meta-analyses. Scand. J. Med. Sci. Sport. 2020; 816-27.

4. Nimwegen M Van, Speelman AD, Hofman-Van Rossum EJM, Overeem S, Deeg DJH, Borm GF, et al. Physical inactivity in Parkinson's disease. J Neurol. 2011; 258(12):2214-21.

5. Fancourt, D., and Finn, S. (2019). What is the evidence on the role of the arts in improving health and well-being? A scoping review. WHO Regional Office Europe. Available online at: http://www.euro.who.int/en/publications/abstracts/what-is-the-evidence-on-the-role-of-the-arts-inimproving-health-and-well-being-a-scoping-review-2019.

6. Fernández-Argüelles EL, Rodríguez-Mansilla J, Antunez LE, Garrido-Ardila EM, Muñoz RP. Effects of dancing on the risk of falling related factors of healthy older adults: A systematic review. Arch. Gerontol. Geriatr. 2015; $1-8$.

7. Mattle M, Chocano-Bedoya PO, Fischbacher M, Meyer U, Abderhalden LA, Lang W, et al. Association of Dance-Based Mind-Motor Activities with Falls and Physical Function among Healthy Older Adults: A Systematic Review and Meta-analysis. JAMA Netw Open. American Medical Association; 2020;3:2017688.

8. Woei-Ni Hwang P, Braun KL. The effectiveness of dance interventions to improve older adults' health: A systematic literature review. Altern. Ther. Health Med. 2015; 64-70.

9. Kshtriya S, Barnstaple R, Rabinovich DB, DeSouza JFX. Dance and Aging: A Critical Review of Findings in Neuroscience. Am. J. Danc. Ther. 2015; 37(2): 81-112.

10. McNeely ME, Duncan RP, Earhart GM. A comparison of dance interventions in people with Parkinson disease and older adults. Maturitas. 2015;81:10-6. 
11. Meng X, Li G, Jia Y, Liu Y, Shang B, Liu P, et al. Effects of dance intervention on global cognition, executive function and memory of older adults: a meta-analysis and systematic review. Aging Clin. Exp. Res. 2020; 7-19.

12. dos Santos Delabary M, Komeroski IG, Monteiro EP, Costa RR, Haas AN. Effects of dance practice on functional mobility, motor symptoms and quality of life in people with Parkinson's disease: a systematic review with meta-analysis. Aging Clin. Exp. Res. 2018; 727-35.

13. Kalyani HHN, Sullivan K, Moyle G, Brauer S, Jeffrey ER, Roeder L, et al. Effects of Dance on Gait, Cognition, and Dual-Tasking in Parkinson's Disease: A Systematic Review and Meta-Analysis. J. Parkinsons. Dis. 2019; 9(2):335-49.

14. Sharp K, Hewitt J. Dance as an intervention for people with Parkinson's disease: A systematic review and meta-analysis. Neurosci Biobehav Rev. 2014; 47:445-56.

15. Carapellotti AM, Stevenson R, Doumas M. The efficacy of dance for improving motor impairments, non-motor symptoms, and quality of life in parkinson's disease: A systematic review and meta-analysis. PLoS One. 2020; 15(8): e0236820.

16. Bek J, Arakaki AI, Lawrence A, Sullivan M, Ganapathy G, Poliakoff E. Dance and Parkinson's: A review and exploration of the role of cognitive representations of action. Neurosci. Biobehav. Rev. 2020; 109: 16-28.

17. Dhami P, Moreno S, DeSouza JFX. New framework for rehabilitation - fusion of cognitive and physical rehabilitation: the hope for dancing. Front Psychol. 2015;5.

18. Rehfeld K, Lüders A, Hökelmann A, Lessmann V, Kaufmann J, Brigadski T, et al. Dance training is superior to repetitive physical exercise in inducing brain plasticity in the elderly. PLoS One. 2018;13:e0196636.

19. Morrow-Howell N, Galucia N, Swinford E. Recovering from the COVID-19 Pandemic: A Focus on Older Adults. J Aging Soc Policy. 2020;32:526-35.

20. Wu B. Social isolation and loneliness among older adults in the context of COVID-19: a global challenge. Glob Heal Res Policy. 2020;5:1-3.

21. Chen AT, Ge S, Cho S, Teng AK, Chu F, Demiris G, et al. Reactions to COVID-19, information and technology use, and social connectedness among older adults with pre-frailty and frailty. Geriatr Nurs. 2021;42:188-95.

22. Schirinzi T, Di Lazzaro G, Salimei C, Cerroni R, Liguori C, Scalise S, et al. Physical Activity Changes and Correlate Effects in Patients with Parkinson's Disease during COVID-19 Lockdown. Mov Disord Clin Pract. 2020; 7(7):797-802.

23. Quinn L, MacPherson C, Long K, Shah H. Promoting physical activity via telehealth in people with Parkinson disease: The path forward after the COVID-19 pandemic? Phys Ther. 2020; 100:1730-1736.

24. Hülür G, Macdonald B. Rethinking social relationships in old age: Digitalization and the social lives of older adults. Am Psychol. American Psychological Association Inc.; 2020;75:554-66.

25. Afshari M, Yang A, Bega D. Motivators and Barriers to Exercise in Parkinson's Disease. J Park Dis. 2017;7:703-11. 
26. Ellis T, Boudreau JK, DeAngelis TR, Brown LE, Cavanaugh JT, Earhart GM, et al. Barriers to exercise in people with parkinson disease. Phys Ther. 2013;93:628-36.

27. Bek J, Groves M, Leventhal D, Poliakoff E. Dance at home for people with Parkinson's during COVID19 and beyond: Participation, perceptions, and prospects. Front Neurol. 2021; 12.

28. R Core Team 2021. R A Lang. Environ. Stat. Comput. 2021.

29. Bates D, Maechler M, Bolker B, Walker S. Package"lme4". J Stat Softw. 2015;

30. Braun V, Clarke V. Using thematic analysis in psychology. Qual Res Psychol. 2006; ;3(2):77-101.

31. Rocha PA, Slade SC, McClelland J, Morris ME. Dance is more than therapy: Qualitative analysis on therapeutic dancing classes for Parkinson's. Complement Ther Med. 2017;34.

32. Houston S, McGill A. A mixed-methods study into ballet for people living with Parkinson's. Arts Health. 2013;5:103-19.

33. Bek J, Arakaki A, Derbyshire-Fox F, Ganapathy G, Sullivan M, Poliakoff E. More than Movement: Exploring motor simulation, creativity, and function in co-developed dance for Parkinson's. Front Psychol. 2022.

34. Fontanesi C, DeSouza JF. Beauty That Moves: Dance for Parkinson's Effects on Affect, Self-Efficacy, Gait Symmetry, and Dual Task Performance. Front Psychol. 2021; 11:3896.

35. Hackney ME, Earhart GM. Effects of dance on gait and balance in Parkinsons disease: A comparison of partnered and nonpartnered dance movement. Neurorehabil Neural Repair. 2010;24:384-92.

36. Kunkel D, Robison J, Fitton C, Hulbert S, Roberts L, Wiles R, et al. It takes two: the influence of dance partners on the perceived enjoyment and benefits during participation in partnered ballroom dance classes for people with Parkinson's. Disabil Rehabil. 2018; 40(16), 1933-1942.

37. Ashworth NL, Chad KE, Harrison EL, Reeder BA, Marshall SC. Home versus center based physical activity programs in older adults. Cochrane Database Syst Rev. 2005; 1.

38. Kelly MP, Leventhal D. Dance as Lifeline: Transforming Means for Engagement and Connection in Times of Social Isolation. Health Promot Pract. 2021;22:64S-69S.

39. Silva PA. Are We Ready to Dance at Home?: A Review and Reflection of Available Technologies. International Conference on Human-Computer Interaction. 2019; 216-231.

40. Dos Santos A, Pegollo F, Alencar R, Avanzi R, Pompeu JE. A new tool for assessment and balance training of patients with Parkinson's disease based on low cost comercial Wii balance board. Movement Disorders. 2012;27: S301-S301.

41. Lee NY, Lee DK, Song HS. Effect of virtual reality dance exercise on the balance, activities of daily living, and depressive disorder status of Parkinson's disease patients. J Phys Ther Sci. 2015; 27(1):145-147. 